\title{
免疫金铂纳米合金催化共振散射光谱法测定痕量人线毛膜促性腺激素
}

\author{
蒋治良* 姚东梅李芳梁爱惠* \\ (药用资源化学与药物分子工程教育部重点实验室 珍稀濒危动植物生态与环境保护省部共建教育部重点实验室 \\ 广西师范大学 桂林 541004)
}

\begin{abstract}
摘要 以 $\mathrm{NaBH}_{4}$ 为还原剂, 制备了金与铂物质的量比为 $49: 1$ 的金铂纳米合金(GP). 用兔抗人线毛膜促性腺激素抗体 (RhCG) 修饰 $\mathrm{AuPt}$ 获得了免疫纳米合金探针 (GP-RhCG). 在 $\mathrm{pH} 5.8$ 磷酸氢二钠-柠檬酸缓冲溶液及 $\mathrm{KCl}$ 存在的条件下, GP-RhCG 探针发生非特异性聚集, 在 $590 \mathrm{~nm}$ 处有一个较强的共振散射峰. 当有人线毛膜促性腺激素 $(\mathrm{hCG})$ 存在时, 聚 集的 GP-RhCG 探针与 hCG 发生特异性结合, 生成分散性较好的 GP-RhCG-hCG 免疫复合物, 导致 $590 \mathrm{~nm}$ 处共振散射 峰强度降低. 其共振散射峰强度降低值 $\Delta I_{590} \mathrm{~nm}$ 与 $\mathrm{hCG}$ 浓度在 $6.67 \sim 86.7 \mathrm{ng} / \mathrm{mL}$ 范围内呈现良好线性关系. 免疫反应液 中形成的 GP-RhCG-hCG 免疫复合物对葡萄糖-铜(II)体系具有较强的催化作用, 其产物在 $610 \mathrm{~nm}$ 处有一较强共振散射 峰. 随着 hCG 浓度增大, 形成的 GP-RhCG-hCG 复合物越多, 其催化作用增强, $610 \mathrm{~nm}$ 处的共振散射峰增强. 其共振散 射峰增大值 $\Delta I_{610 \mathrm{~nm}}$ 与 $\mathrm{hCG}$ 浓度在 $3.33 \sim 133 \mathrm{ng} / \mathrm{mL}$ 范围内呈线性关系.
\end{abstract}

关键词 人线毛膜促性腺激素; 金铂纳米合金; 免疫纳米催化; 共振散射光谱法

\section{Resonance Scattering Assay for Trace Human Chorionic Gonadotrophin Using Gold-platinum Nanoalloy Immunoprobe as Catalyst}

\author{
Jiang, Zhiliang* Yao, Dongmei Li, Fang Liang, Aihui* \\ (Key Laboratory for the Chemistry and Molecular Engineering of Medicinal Resources, Education Ministry; \\ Key Laboratory of Ecology of Rare and Endangered Species and Environmental Conservation of Education Ministry,
}

Guangxi Normal University, Guilin 541004)

\begin{abstract}
In the presence of sodium citrate as stabilizer, a gold-platinum nanoalloy (GP) in the mole ratio of $49: 1$ was prepared by using $\mathrm{NaBH}_{4}$ as a reducing agent. The rabbit anti-human chorionic gonadotrophin antibody (RhCG) was labeled by the GP to obtain a stable immune GP probe (GP-RhCG) for the human chorionic gonadotrophin (hCG). In a pH 5.8 $\mathrm{NaH}_{2} \mathrm{PO}_{4}$-citric acid buffer solution and in the presence of $\mathrm{KCl}$, the GP-RhCG probe was aggregated un-specifically to big particles that exhibited a resonance scattering (RS) peak at $590 \mathrm{~nm}$. When the hCG was added, the GP-RhCG probe combined specifically with the hCG to form a dispersed GP-RhCG-hCG immunocomplex that resulted in the RS intensity decreasing at $590 \mathrm{~nm}$. The decreased RS intensity $\left(\Delta I_{590 \mathrm{~nm}}\right)$ is linear with hCG concentration in the range of $6.67-86.7 \mathrm{ng} / \mathrm{mL}$, with a detection limit of $3.7 \mathrm{ng} / \mathrm{mL}$ hCG. The immunocomplex exhibited a strong catalytic effect on the slow $\mathrm{Cu}_{2} \mathrm{O}$ particle reaction of glucose- $\mathrm{Cu}(\mathrm{II})$, and the particles appeared a RS peak at $610 \mathrm{~nm}$. When the hCG concentration increased in the immune reaction system, the immunocomplex concentration increased, and the catalytic product of $\mathrm{Cu}_{2} \mathrm{O}$ particles increased, which caused the enhancement of RS intensity at $610 \mathrm{~nm}$. The increased intensity $\left(\Delta I_{610 \mathrm{~nm}}\right)$ is linear with the hCG concentration in the range of $3.33-133 \mathrm{ng} / \mathrm{mL}$, with a detection limit of $1.7 \mathrm{ng} / \mathrm{mL} \mathrm{hCG}$. In addition, the influence of foreign substances on the determination of hCG was examined. The results show that 150 times of $L$-aspartic acid, urea, BSA, glycine, $\mathrm{Ca}(\mathrm{II})$ and $\mathrm{Zn}(\mathrm{II}), 50$ times of $L$-lysine and $L$-cysteine, 30 times of IgG and IgA, 40 times of IgM did not interfered with the determination of $80 \mathrm{ng} / \mathrm{mL}$ hCG. This indicates that this method has good selectivity. The hCG content in the serum sample was analyzed by the GP RS method and GP-catalytic RS method, the results are agreement with those of the chemiluminescence experiments. Both RS methods are of some advantages including low-cost, simplicity, rapidity, high sensitivity and selectivity.

Keywords human chorionic gonadotrophin; gold-platinum nanoalloy; immunonanocatalysis; resonance scattering assay
\end{abstract}

* E-mail: zljiang@mailbox.gxnu.edu.cn; ahliang2008@163.com

Received March 30, 2012; published May 26, 2012.

Project supported by the National Natural Science Foundation of China (Nos. 21165005, 21075023) and the Fund of Key Laboratory for the Chemistry and Molecular Engineering of Medicinal Resources (Guangxi Normal University), Ministry of Education of China (No. CMEMR2011-10).

项目受国家自然科学基金(Nos. 21165005, 21075023)和药用资源化学与药物分子工程教育部重点实验室基金(No. CMEMR2011-10)资助. 


\section{1 引言}

人线毛膜促性腺激素 $(\mathrm{hCG})$, 是由孕妇胎盘合体的 滋养层细胞分泌的一种糖蛋白, 由 $\alpha$-和 $\beta$-两个亚单位构 成, 它是妊娠的重要诊断标记 ${ }^{[1 \sim 3]}$ 以及最重要的肿瘤标 记物之一 ${ }^{[4]}$. 健康女性在血清或尿液中的 hCG 含量一般 低于 $14.3 \mathrm{mIU} / \mathrm{mL}$, 如果 $\mathrm{hCG}$ 的含量升高, 则有可能是 怀孕或是与滋养层的疾病有关. 因此, 对 $\mathrm{hCG}$ 进行定量 检测具有重要的临床意义. 目前, hCG 的检测方法主要 有电化学免疫分析法 ${ }^{[4]}$ 、电化学发光免疫分析法 ${ }^{[8]}$ 、 非标记安培免疫分析法 ${ }^{[9]}$ 、化学发光免疫分析法 ${ }^{[10,11]}$ 、 光致发光法 ${ }^{[12]}$ 、荧光免疫分析法 ${ }^{[13,14]}$ 、共振散射光谱

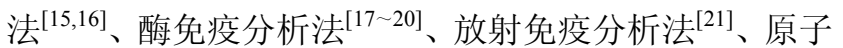
发射法 ${ }^{[22]}$ 等. 这些方法当中, 电化学免疫分析法操作简 单方便、稳定; 苂光免疫分析法简单、快速, 但在实际 检测分析中存在本底荧光的干扰; 酶免疫分析比放射免 疫分析更加灵敏, 简便灵活, 但酶价格较贵; 放射免疫 分析法灵敏度高、特异性强、应用广泛，有放射线辐射 和污染的可能; 化学发光灵敏度较高、检测快速, 但操 作步骤较为复杂. 共振散射光谱法具有灵敏、快速、简 便等特点, 已应用于痕量抗原的免疫测定 ${ }^{[23]}$. 基于纳米 金的生物相容性和共振散射效应, 发展了灵敏度较高的 免疫纳米金和适配体纳米金共振散射光谱分析法 ${ }^{[24 ~ 29]}$. 将纳米金标免疫反应与纳米金催化反应结合, 建立了高 灵敏高选择性的免疫纳米金催化共振散射光谱法 ${ }^{[30,31]}$. 本文用 $\mathrm{GP}$ 标记 $\mathrm{hCG}$ 抗体获得了 $\mathrm{hCG}$ 免疫纳米金探针, 将纳米合金标记免疫反应与葡萄糖-铜(II)纳米催化反 应结合起来, 无需离心分离, 建立了简便快速灵敏检测 $\mathrm{hCG}$ 的共振散射光谱方法.

\section{2 结果与讨论}

\section{1 方法原理}

在 $\mathrm{pH} 5.8$ 磷酸氢二钠-柠檬酸缓冲溶液及 $\mathrm{KCl}$ 存在 的条件下, GP-RhCG 探针发生非特异性聚集, 溶液颜色 为紫色, 在 $590 \mathrm{~nm}$ 处有一个较强的共振散射峰. 随着人 线毛膜促性腺激素(hCG)浓度的增加, 聚集的 GP-RhCG 探针与 $\mathrm{hCG}$ 发生特异性结合, 生成分散性较好的 GP-RhCG-hCG 免疫复合物, 溶液颜色由紫色变为红色, 导致 $590 \mathrm{~nm}$ 处共振散射峰线性降低. 据此建立了一个 简便快速测定 $\mathrm{hCG}$ 的共振散射光谱法(图 1).

在没有纳米催化剂时，葡萄糖-铜(II)反应很慢，共 振散射值较低. GP, GP-RhCG, GP-RhCG-hCG 对葡萄 糖-铜(II)反应均具有较强的催化作用, 生成的 $\mathrm{Cu}_{2} \mathrm{O}$ 微 粒存在共振散射效应; 而聚集的 GP-RhCG 探针和聚集 的 GPA 的催化作用较弱. 因此, 可免除分离探针聚集体 与探针的免疫复合物. 随着 $\mathrm{hCG}$ 浓度增大, 形成分散的 GP-RhCG-hCG 复合物越多, 其催化作用增强, $610 \mathrm{~nm}$ 处的共振散射峰线性增大. 据此可建立一个灵敏的测定 $\mathrm{hCG}$ 的纳米金铂催化共振散射光谱分析法(图 1).
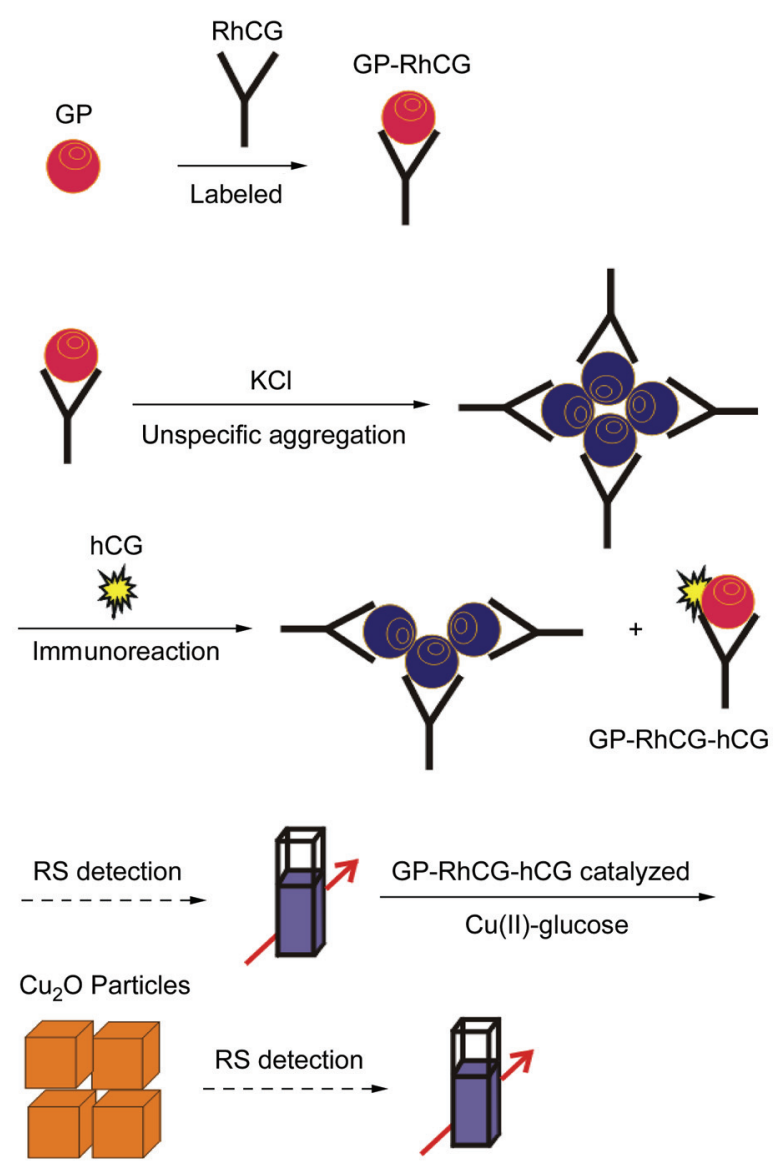

图 1 免疫金铂纳米合金催化共振散射光谱测定 $\mathrm{hCG}$ 原理

Figure 1 Principle of detection hCG using GP catalytic RS method

\section{2 共振散射光谱}

在一定浓度 $\mathrm{KCl}$ 溶液中, 金铂纳米粒子发生聚集, 在 $590 \mathrm{~nm}$ 处产生一个较强的共振散射峰. 用兔抗 hCG 标记金铂纳米合金, 其共振散射光强度较弱. 在磷酸氢 二钠-柠檬酸缓冲液及有较高浓度盐 $\mathrm{KCl}$ 存在时, 金铂 标记兔抗 $\mathrm{hCG}$ 发生非特异性聚集, 溶液颜色由红色变 为紫色, 也在 $590 \mathrm{~nm}$ 处产生一个较强的共振散射峰. 随 着 $\mathrm{hCG}$ 浓度的增加, 金铂标记兔抗 $\mathrm{hCG}$ 与 $\mathrm{hCG}$ 发生特 异性结合，生成分散的免疫复合物增多，溶液颜色由紫 色变为红色, $590 \mathrm{~nm}$ 处的共振散射峰降低(图 2). 本文选 择 $590 \mathrm{~nm}$ 测定.

GP-RhCG 对葡萄糖-铜(II)反应具有催化作用, 生 成的氧化亚铜微粒在 $610 \mathrm{~nm}$ 处有一个较强共振散射峰. 随着 $\mathrm{hCG}$ 浓度的增加，反应液中分散的 GP-RhCG-hCG 催化剂增多, 生成的 $\mathrm{Cu}_{2} \mathrm{O}$ 微粒增加, $610 \mathrm{~nm}$ 的共振散 射峰强度线性增强(图 3). 本文选择 $610 \mathrm{~nm}$ 测定 hCG.

\section{3 吸收光谱}

图 4 表明，红色的金纳米粒子( $\mathrm{Au}$ )在 $512 \mathrm{~nm}$ 处有一 个表面等离子体共振吸收峰; 红色的金铂纳米粒子也在 $512 \mathrm{~nm}$ 处有一个表面等离子体共振吸收峰, 说明形成 了金铂纳米合金. 当 $\mathrm{KCl}$ 与金铂纳米合金发生聚集反应 


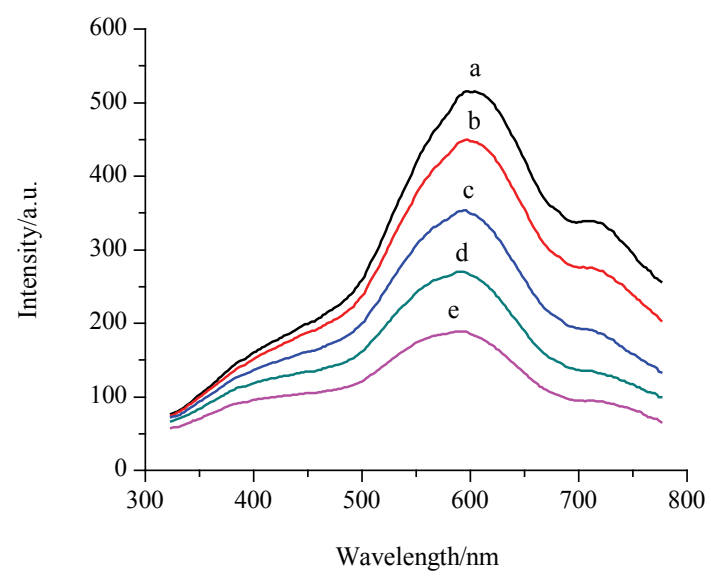

图 2 GP-RhCG-hCG 体系的共振散射光谱

Figure 2 RS spectra of GP-RhCG-hCG systems

(a) $8.48 \mu \mathrm{g} / \mathrm{mL}$ GP-RhCG-pH $5.8 \mathrm{Na}_{2} \mathrm{HPO}_{4}$-citric acid- $1.0 \mathrm{mg} / \mathrm{L} \mathrm{KCl}$; (b) a+ $13.3 \mathrm{ng} / \mathrm{mL}$ hCG; (c) a $+33.3 \mathrm{ng} / \mathrm{mL}$ hCG; (d) a $+53.3 \mathrm{ng} / \mathrm{mL}$ hCG; (e) a + $86.7 \mathrm{ng} / \mathrm{mL}$ hCG

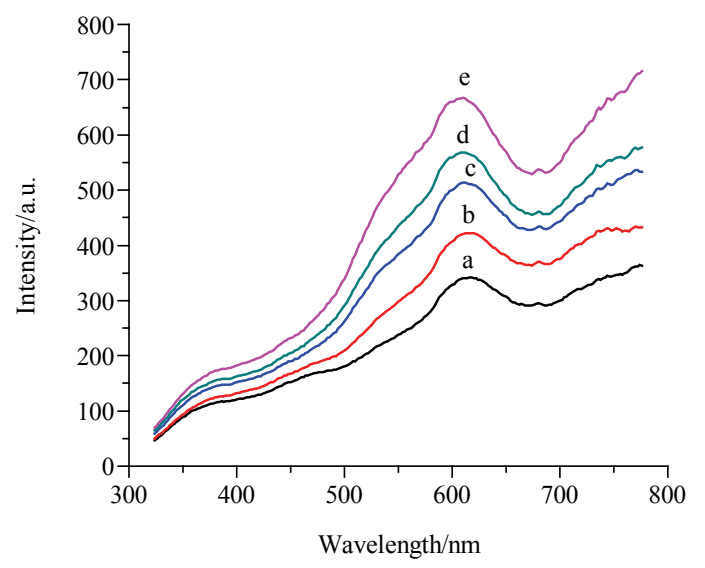

图 3 葡萄糖-铜(II)-GP-RhCG-hCG 催化体系的共振散射光谱 Figure 3 RS spectra of glucose-Cu(II)-GP-RhCG-hCG systems (a) $2.0 \mathrm{mmol} / \mathrm{L} \mathrm{CuSO} \mathrm{L}_{4}-61.5 \mathrm{mmol} / \mathrm{L} \mathrm{KNaC} \mathrm{KH}_{4} \mathrm{O}_{6}-0.37 \mathrm{mmol} / \mathrm{L}$ glucose- 150 $\mu \mathrm{L}$ reaction solution; (b) $8 \mathrm{ng} / \mathrm{mL}$ hCG; (c) $50 \mathrm{ng} / \mathrm{mL}$ hCG; (d) $150 \mathrm{ng} / \mathrm{mL}$ hCG; (e) $200 \mathrm{ng} / \mathrm{mL} \mathrm{hCG}$

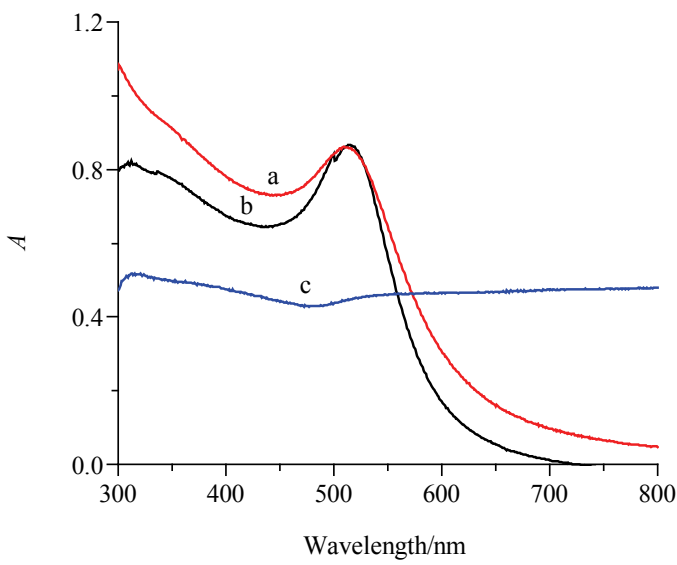

图 4 GP 体系的吸收光谱

Figure 4 Absorption spectra of GP systems

(a) $56.0 \mu \mathrm{g} / \mathrm{mL} \mathrm{GP}$; (b) $57.1 \mu \mathrm{g} / \mathrm{mL} \mathrm{Au}$; (c) $44.8 \mu \mathrm{g} / \mathrm{mL}$ GPA
后，溶液颜色由深红色变成蓝紫色，表面等离子体共振 吸收峰消失，因形成较大粒径聚集微粒的散射作用，在 可见光范围的吸光度趋于一致(图 4c).

当金铂纳米合金用兔抗 $\mathrm{hCG}$ 标记后，其颜色仍为 红色, 在 $512 \mathrm{~nm}$ 处有一个表面等离子体共振吸收峰(图 $5 a)$. 在磷酸氢二钠一柠檬酸缓冲液及 $\mathrm{KCl}$ 存在条件下, 金铂标记兔抗 hCG 发生非特异性聚集, 溶液颜色为紫 色, 在 $530 \mathrm{~nm}$ 处有一个吸收峰, 与 GP-RhCG 相比, 峰 发生红移, 峰变宽且在 $600 \mathrm{~nm}$ 以上波长的吸收值增大, 这说明体系形成了较大粒径的聚集体. 随着 $\mathrm{hCG}$ 浓度 增大, GP-RhCG 与 hCG 发生特异性结合, 溶液颜色由紫 色变为红色, 但在 $530 \mathrm{~nm}$ 处的吸收峰稍有降低. 这是由 于 $(\mathrm{GP}-\mathrm{RhCG})_{n}$ 聚集体浓度降低和分散的 GP-RhCG-hCG 浓度增大, 且二者均为大粒径微粒产生较强的散射所 致.

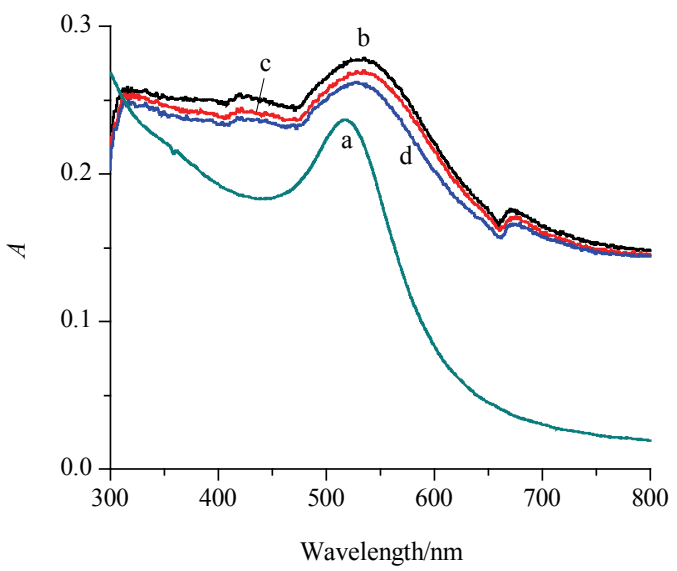

图 5 GP-RhCG-hCG 体系的吸收光谱

Figure 5 Absorption spectra of GP-RhCG-hCG systems

(a) $8.48 \mu \mathrm{g} / \mathrm{mL}$ GP-RhCG; (b) $8.48 \mu \mathrm{g} / \mathrm{mL}$ GP-RhCG-pH $5.8 \mathrm{Na}_{2} \mathrm{HPO}_{4}$-citric acid-1.0 mg/L KCl; (c) b+26.7 ng/mL hCG; (d) b+60.0 ng/mL hCG

\section{4 免疫反应条件的优化}

兔抗 $\mathrm{hCG}$ 修饰纳米金与 $\mathrm{hCG}$ 免疫反应已用于共振 散射光谱分析 ${ }^{[9]}$ ，随着 $\mathrm{hCG}$ 浓度增大其共振散射信号线 性降低，但其灵敏度不高. 本实验结果表明，用金铂纳 米合金较纳米金效果更好. 图 6 给出了 3 种不同物质的 量比金铂纳米合金测定 $\mathrm{hCG}$ 的关系曲线. 其中 $\mathrm{Au}: \mathrm{Pt}$ 物质的量比为 $49: 1$ 的最好, 故选择此纳米合金进行后 续研究.

考察了 $\mathrm{pH}$ 值对免疫反应 $\Delta I_{590 \mathrm{~nm}}$ 的影响. 当 $\mathrm{pH}$ 值 为 5.8 时, $\Delta I_{590 \mathrm{~nm}}$ 具有最大值. 因此本文选择 $\mathrm{pH} 5.8$ 的 $\mathrm{Na}_{2} \mathrm{HPO}_{4}$-柠檬酸缓冲液. 还考察了 $\mathrm{Na}_{2} \mathrm{HPO}_{4}$-柠檬酸缓 冲液浓度(以 $\mathrm{Na}_{2} \mathrm{HPO}_{4}$ 浓度计)对 $\Delta I_{590 \mathrm{~nm}}$ 的影响. 在 $\mathrm{pH}$ 值 5.8 的 $\mathrm{Na}_{2} \mathrm{HPO}_{4}$-柠檬酸缓冲液中, GP-RhCG 发生聚 集，且缓冲溶液浓度越大，其聚集程度越大， $I_{590 \mathrm{~nm}}$ 值越 大. 当 $\mathrm{Na}_{2} \mathrm{HPO}_{4}$-柠檬酸缓冲液浓度达到 $20.2 \mathrm{mmol} / \mathrm{L}$ 时, $\Delta I_{590 \mathrm{~nm}}$ 最大. 故本文选择 $20.2 \mathrm{mmol} / \mathrm{L} \mathrm{Na}_{2} \mathrm{HPO}_{4}$-柠檬酸 


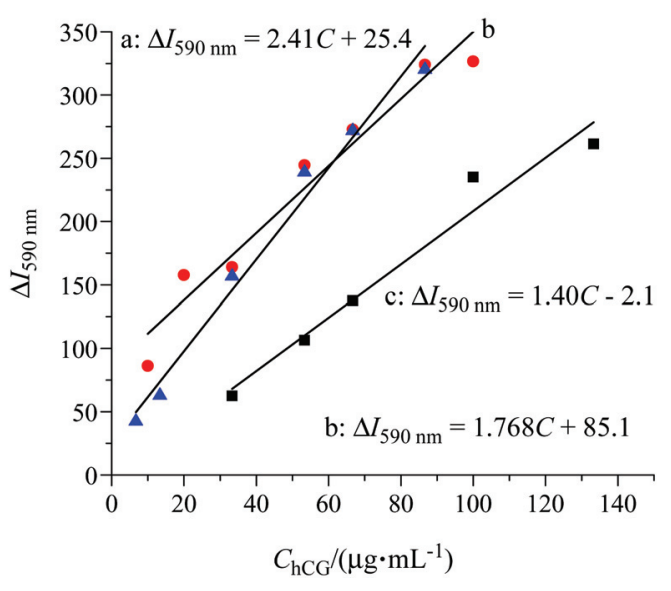

图 6 不同物质的量比金铂纳米合金体系 $\Delta I_{590 \mathrm{~nm}}$ 与 $\mathrm{hCG}$ 浓度的关系 Figure 6 Relationship between hCG concentration and $\Delta I_{590 \mathrm{~nm}}$ for the GP system.

(a) GP (49:1); (b) GP (32:1); (c) GP (19:1)

缓冲液. $\mathrm{KCl}$ 的加入可以使 $\mathrm{GP}-\mathrm{RhCG}$ 更好地聚集, 提高 了方法的灵敏度, 本文选择 $0.1 \% \mathrm{KCl}$ 溶液. GP-RhCG 对 免疫反应的影响如图 7. 当 GP-RhCG 浓度为 $8.48 \mu \mathrm{g} / \mathrm{mL}$ 时, $\Delta I_{590 \mathrm{~nm}}$ 趋于平衡. 因此本文选择 $8.48 \mu \mathrm{g} / \mathrm{mL}$ 作为 GP-RhCG 的最佳用量.

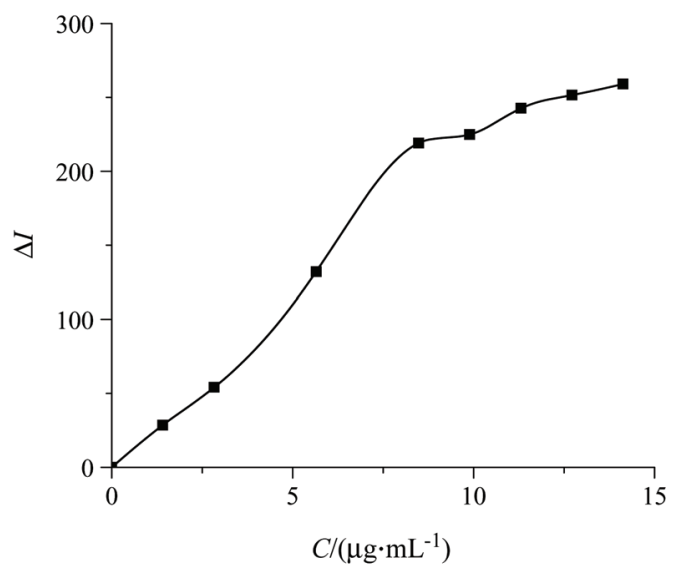

图 7 GP-RhCG 浓度的影响

Figure 7 Effect of GP-RhCG concentration on $\Delta I_{590 \mathrm{~nm}}$ pH $5.8 \mathrm{Na}_{2} \mathrm{HPO}_{4}$-citric acid-1.0 mg/L KCl-66.7 ng/mL hCG

\section{5 催化反应条件的优化}

催化反应可以放大信号, 无机催化反应、酶催化反 应在分析化学中已有应用. 近年来, 纳米粒子的催化作 用被发现并用于生化分析. 在共振散射光谱分析中, 一 个较好的纳米催化反应应具备灵敏、空白值低, 反应易 控制且生成的微粒产物稳定等. 与 $\mathrm{Ag}(\mathrm{I})$-hydroquinone 和 $\mathrm{Cu}(\mathrm{II})-\mathrm{N}_{2} \mathrm{H}_{4}$ 等纳米催化体系比较 ${ }^{[16,31]}$, 葡萄糖-菲林 试剂体系是一个较好的体系. 在没有催化剂的常温条件 下, 葡萄糖-菲林试剂反应很难发生, 共振散射值较低. 铂纳米粒子的催化作用较弱且不稳定. 金纳米粒子和该
金铂纳米合金均具有较强的催化作用，但金纳米粒子的 免疫反应不及金铂纳米粒子的灵敏, 故选择金铂纳米粒 子标记抗体. 当有金铂纳米催化剂 GP 存在时, 葡萄糖 还原铜(II)生成 $\mathrm{Cu}_{2} \mathrm{O}$ 微粒; 但聚集的 $\mathrm{GP}$ 微粒(GPA)的催 化作用很弱, 体系共振散射强度较弱. 因此, 无需进行 离心分离 GP 与 GPA. 图 8 表明, 抗体吸附在纳米合金 表面降低了其催化活性, 但 GP-RhCG 仍具有很强的催 化作用. 本文分别考察了 $\mathrm{CuSO}_{4} 、 \mathrm{KNaC}_{4} \mathrm{H}_{4} \mathrm{O}_{6}-\mathrm{NaOH}$ 、 葡萄糖、温度、时间对该体系 $\Delta I_{610 \mathrm{~nm}}$ 的影响，当采用 $2.0 \mathrm{mmol} / \mathrm{L} \mathrm{CuSO}_{4} 、 61.5 \mathrm{mmol} / \mathrm{L} \mathrm{KNaC}_{4} \mathrm{H}_{4} \mathrm{O}_{6}-\mathrm{NaOH}$ 、 $0.37 \mathrm{mmol} / \mathrm{L}$ 葡萄糖、 $70{ }^{\circ} \mathrm{C} 、 7 \mathrm{~min}$ 条件时, $\Delta I_{610 \mathrm{~nm}}$ 达 到最大值, 本文采用上述条件进行催化测定 hCG. 还考 察了免疫反应液对体系的影响. 当免疫反应液用量为 $150 \mu \mathrm{L}$ (稀释 50 倍)时, $\Delta I_{610 \mathrm{~nm}}$ 具有最大值. 因此本文选 用 $150 \mu \mathrm{L}$ 稀释 50 倍的免疫反应液作为催化液.

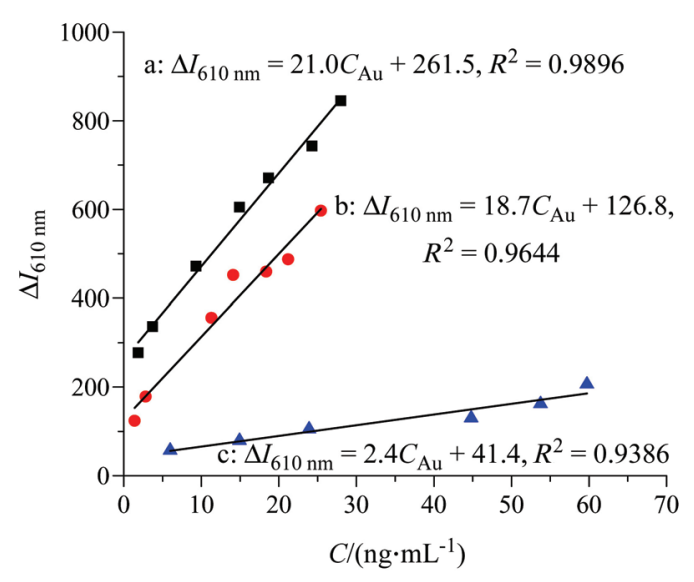

图 8 纳米粒子浓度与 $\Delta I_{610 \mathrm{~nm}}$ 的关系

Figure 8 Relationship between nanoparticle concentration and $\Delta I_{610 \mathrm{~nm}}$ (a) GP; (b) GP-RhCG; (c) GPA.

\section{6 工作曲线}

在最佳条件下，测量不同含量 $\mathrm{hCG}$ 反应液及其催 化反应的共振散射光强度. 以 $\mathrm{hCG}$ 浓度 $C_{\mathrm{hCG}}$ 对 $\Delta I_{590 \mathrm{~nm}}$ 、 $\Delta I_{610 \mathrm{~nm}}$ 作图绘制工作曲线，其线性范围、线性方程、相 关系数及检出限 $(3 \alpha)$ 分别为 $6.67 \sim 86.7 \mathrm{ng} / \mathrm{mL}, \Delta I_{590 \mathrm{~nm}}=$ $3.62 C+25.4, r=0.9832,3.7 \mathrm{ng} / \mathrm{mL} ; 3.33 \sim 133 \mathrm{ng} / \mathrm{mL}$, $\Delta I_{610 \mathrm{~nm}}=2.04 C+76.7, r=0.9612,1.7 \mathrm{ng} / \mathrm{mL}$. 与已报道 的方法相比较(表1), 本法操作简单, 快速, 灵敏度较高, 使用较常见的仪器且较为廉价, 是目前测定 $\mathrm{hCG}$ 较简 便的方法之一.

\section{7 干扰实验}

按实验方法, 考察了血清中常见的共存物质对本体 系的干扰. 结果表明, 150 倍 $L$-天冬氨酸、尿素、BSA、 氨基乙酸、 $\mathrm{Ca}(\mathrm{II}) 、 \mathrm{Zn}(\mathrm{II}), 50$ 倍 $L$-赖氨酸、 $L$-半胱氨酸, 30 倍 IgG、IgA, 40 倍 IgM 等对 $80 \mathrm{ng} / \mathrm{mL}$ hCG 测定不影 响. 说明本方法具有较好的选择性. 
表 1 hCG 分析方法的比较

Table 1 Comparing of the reported assays for hCG

\begin{tabular}{|c|c|c|c|c|c|}
\hline Assay & Principle & Linear range & DL & Comment & Ref. \\
\hline 电化学免疫分析法 & $\begin{array}{l}\text { anti-hCG 修饰到碳纳米管上, 蛋白质化学 } \\
\text { 行为的变化引起电化学信号变换 }\end{array}$ & $10 \sim 10000 \mathrm{ng} / \mathrm{mL}$ & $8 \mathrm{ng} / \mathrm{mL}$ & 稳定, 但灵敏度不高 & [7] \\
\hline $\begin{array}{l}\text { 电化学发光免疫分析 } \\
\text { 法 }\end{array}$ & $\begin{array}{l}\text { 金纳米粒子与 } \mathrm{Ru}(\mathrm{bpy})_{3}{ }^{2+}-\mathrm{TiO}_{2} \text { 涂层及抗 } \\
\text { 体抗原组成三明治结构, 检测 ECL 信号 }\end{array}$ & $2 \sim 2500 \mathrm{ng} / \mathrm{mL}$ & $0.7 \mathrm{ng} / \mathrm{mL}$ & 灵敏度高, 但方法复杂 & {$[8]$} \\
\hline $\begin{array}{l}\text { 非标记安培免疫分析 } \\
\text { 法 }\end{array}$ & $\begin{array}{l}\mathrm{hCG} \text { 固定在纳米多孔金上, 氢醌为氧化 } \\
\text { 还原引物, 电流变化与 } \mathrm{hCG} \text { 有关 }\end{array}$ & $0.5 \sim 40 \mathrm{ng} / \mathrm{mL}$ & $0.034 \mathrm{ng} / \mathrm{mL}$ & 较准确 & {$[9]$} \\
\hline 光致发光法 & $\begin{array}{l}\text { 抗体 } \mathrm{hCG} \text { 结合到 } \mathrm{ZnO} \text { 阵列上, 导致 } \mathrm{ZnO} \\
\text { 阵列 } \mathrm{PL} \text { 增强 }\end{array}$ & $2 \sim 20 \mathrm{ng} / \mathrm{mL}$ & - & 灵敏度高, 但不太稳定 & [12] \\
\hline 苂光免疫分析法 & 阳离子交换树脂毛细管柱的流动免疫分析 & $2.5 \sim 150 \mu \mathrm{g} / \mathrm{mL}$ & - & 简单, 快速 & [13] \\
\hline 酶免疫分析法 & $\begin{array}{l}\mathrm{hCG} \text { 与 POD 标记的 anti-hCG 直接通过凝 } \\
\text { 胶过滤的 HPLC 分析 }\end{array}$ & $0 \sim 20 \mu \mathrm{g} / \mathrm{mL}$ & $100 \mathrm{ng} / \mathrm{mL}$ & 简便 & {$[17]$} \\
\hline 原子发射法 & $\begin{array}{l}\text { 银纳米粒子标记的液态电极等离子体原 } \\
\text { 子发射光谱法 }\end{array}$ & $0.01 \sim 1 \mathrm{ng} / \mathrm{mL}$ & $1.3 \mathrm{pg} / \mathrm{mL}$ & 灵敏, 成本高 & [22] \\
\hline 催化共振散射法 & $\begin{array}{l}\mathrm{Au} \text { 标记的 anti-hCG 与 } \mathrm{hCG} \text { 发生免疫反 } \\
\text { 应, 用离心液催化 } \mathrm{Ag}(\mathrm{I}) \text {-hydroquinone }\end{array}$ & $0.25 \sim 20.8 \mu \mathrm{g} / \mathrm{mL}$ & $83 \mathrm{ng} / \mathrm{mL}$ & 灵敏, 但须离心分离 & [16] \\
\hline 催化共振散射法 & $\begin{array}{l}\mathrm{GP} \text { 标记的 anti-hCG 与 } \mathrm{hCG} \text { 发生免疫反应, } \\
\text { 用免疫反应液催化葡萄糖还原菲林试剂 }\end{array}$ & $3.33 \sim 133 \mathrm{ng} / \mathrm{mL}$ & $1.7 \mathrm{ng} / \mathrm{mL}$ & 简便, 快速, 灵敏 & 本法 \\
\hline
\end{tabular}

\section{8 分析应用}

hCG 的免疫分析方法具有较高的选择性和灵敏度, 可无需分离直接用于血清中 $\mathrm{hCG}$ 含量的测定, 可获得 较好的结果. 采用免疫金铂纳米合金共振散射光谱法和 免疫纳米金铂催化一共振散射光谱法测定血清中 $\mathrm{hCG}$ 含 量, 此二法结果与化学发光法参考值一致(表 2).

表 2 血清分析结果

Table 2 Results for the determination of hCG in sera

\begin{tabular}{cccc}
\hline Sample & $\begin{array}{c}\text { GP-RS results }{ }^{a} / \\
\left(\mathrm{mg} \bullet \mathrm{mL}^{-1}\right)\end{array}$ & $\begin{array}{c}\text { GP Cat-RS results } \\
\left(\mathrm{mg} \bullet \mathrm{mL}^{-1}\right)\end{array}$ & $\begin{array}{c}\text { Reference results/ } \\
\left(\mathrm{mg} \bullet \mathrm{mL}^{-1}\right)\end{array}$ \\
\hline 1 & $3.68 \pm 0.18$ & $3.70 \pm 0.22$ & 3.75 \\
2 & $4.54 \pm 0.12$ & $4.49 \pm 0.16$ & 4.50 \\
3 & $2.49 \pm 0.11$ & $2.63 \pm 0.10$ & 2.55 \\
\hline
\end{tabular}

${ }^{a}$ GP-RS 为免疫金铂纳米合金共振散射光谱法, GP Cat-RS 为免疫纳米金铂 催化-共振散射光谱法.

\section{3 结论}

本文用 GP 标记 RhCG 获得了 GP-RhCG 探针. 基于 GP-RhCG 探针发生非特异性聚集和免疫反应建立了一 个测定 $6.67 \sim 86.7 \mathrm{ng} / \mathrm{mL} \mathrm{hCG}$ 的共振散射光谱法. 因 GP-RhCG-hCG 免疫复合物对葡萄糖-铜(II)体系具有较 强的催化作用, 其产物在 $610 \mathrm{~nm}$ 处有一较强共振散射 峰, 据此建立了一个 $3.33 \sim 133 \mathrm{ng} / \mathrm{mL}$ hCG 的免疫纳米 催化一氧化亚铜共振散射光谱法. 前一方法简便快速, 后一方法灵敏且无需离心分离步骤.

\section{4 实验部分}

\section{1 仪器与试剂}

Cary Eclipse 型荧光分光光度计(美国 Varian 公司);
$\mathrm{SK} 1200 \mathrm{H}$ 超声波反应器(上海科导超声仪器有限公司, 工作频率为 $59 \mathrm{kHz}$ ); SYZ-550 型石英亚沸蒸馏水器(江 苏晶玻仪器厂); HH-S2 电热恒温水浴锅(金坛市大地自 动化仪器厂).

\section{2 试剂}

兔抗 $\mathrm{hCG}$ 抗体(北京博奥森生物技术有限公司, Rabbit Anti-Beta-hCG, catalog number bs-0953R): 取 0.2 $\mathrm{mL}$ 抗体稀释液加入到 $0.20 \mathrm{mg}$ 兔抗 $\mathrm{hCG}$ 抗体冻干粉中 混匀, 得到 $0.2 \mathrm{~mL}$ 浓度为 $1 \mathrm{mg} / \mathrm{mL}$ 的 $\mathrm{hCG}$ 抗体浓缩液, 用水稀释至 $10.0 \mathrm{~mL}$, 得到浓度为 $20 \mu \mathrm{g} / \mathrm{mL}$ 的 Rabbit Anti-Beta-hCG (RhCG) 溶液, 于 $4{ }^{\circ} \mathrm{C}$ 保存. 使用时再稀 释 10 倍, 浓度为 $2 \mu \mathrm{g} / \mathrm{mL} .50 \mu \mathrm{g} / \mathrm{mL}$ hCG 抗原标准液(北 京博奥森生物技术有限公司, hCG beta peptides (human), catalog number bs-0953P): 取 $0.50 \mathrm{mg} \mathrm{hCG}$ 冻干粉与 1.0 $\mathrm{mL}$ 水充分混合均匀后, 用水稀释至 $10 \mathrm{~mL}$, 得到 50 $\mu \mathrm{g} / \mathrm{mL} \mathrm{hCG}$ 抗原标准液, 使用前稀释成 $1.0 \mu \mathrm{g} / \mathrm{mL}$ hCG 溶液. pH 5.8 磷酸氢二钠-柠檬酸缓冲溶液: 分别移取 $12.09 \mathrm{~mL} 0.2 \mathrm{~mol} / \mathrm{L} \mathrm{Na}_{2} \mathrm{HPO}_{4}$ 溶液和 $7.91 \mathrm{~mL} 0.1 \mathrm{~mol} / \mathrm{L}$ 柠檬酸溶液到 $20 \mathrm{~mL}$ 的容量瓶中混合均匀, 于 $4{ }^{\circ} \mathrm{C}$ 保存; $10 \% \mathrm{KCl}$ 水溶液: 准确称取 $1.0 \mathrm{~g}$ 氯化钾加水充分溶解 后，用水稀释至 $10 \mathrm{~mL} ; 0.1 \mathrm{~mol} / \mathrm{L} \mathrm{HCl} ; 0.1 \mathrm{~mol} / \mathrm{L} \mathrm{K}_{2} \mathrm{CO}_{3}$; $2.9 \times 10^{-2} \mathrm{~mol} / \mathrm{L} \mathrm{HAuCl}_{4}$ (国药集团化学试剂公司); $2.9 \times 10^{-2} \mathrm{~mol} / \mathrm{L} \mathrm{H}_{2} \mathrm{PtCl}_{6}$ (国药集团化学试剂公司); $0.05 \%$ 硼氢化钠(新配制); $1.0 \%$ 柠檬酸三钠溶液; 菲林 (Fehling) 溶液: $0.2 \mathrm{~mol} / \mathrm{L} \mathrm{CuSO}_{4}$ 溶液, $1.23 \mathrm{~mol} / \mathrm{L}$ 酒石酸 钾钠 $\left(\mathrm{KNaC}_{4} \mathrm{H}_{4} \mathrm{O}_{6}\right)-6.25 \mathrm{~mol} / \mathrm{L} \mathrm{NaOH}$ 溶液; $5.56 \mathrm{mmol} / \mathrm{L}$ 葡萄糖. 所用试剂均为分析纯级, 实验用水为亚沸水.

\section{3 金铂纳米合金的制备}

将 $100 \mathrm{~mL}$ 锥形瓶和大小适合的磁力摚拌子用王水 
$\left(\mathrm{HNO}_{3}: \mathrm{HCl}=1: 3\right.$, 体积比)浸泡 $1 \mathrm{~h}$, 然后用亚沸水洗 净, 烘干. 常温下, 将亚沸水 $40.0 \mathrm{~mL}$ 置于洁净的雉形 瓶内, 在搅拌下依次加入 $490 \mu \mathrm{L} 2.9 \times 10^{-2} \mathrm{~mol} / \mathrm{L}$ $\mathrm{HAuCl}_{4}$ 和 $10 \mu \mathrm{L} 2.9 \times 10^{-2} \mathrm{~mol} / \mathrm{L} \mathrm{H}_{2} \mathrm{PtCl}_{6}$ 溶液, 及 3.5 $\mathrm{mL} 10 \mathrm{mg} / \mathrm{mL}$ 的柠檬酸三钠于雉形瓶中, 搅拌混匀, 向 混合液中缓慢滴加 $3.5 \mathrm{~mL}$ 新配制的 $0.5 \mathrm{mg} / \mathrm{mL} \mathrm{NaBH}_{4}$, 其颜色由无色变紫色最终变为深红色, 继续摚拌 10 $\min$. 用水定容 $50 \mathrm{~mL}$, 得到金铂纳米合金 (GP), 以 $\mathrm{Au}$ 计其浓度为 $56.0 \mu \mathrm{g} / \mathrm{mL}$, 金与铂的摩尔比为 $49: 1$ (以反 应前 $\mathrm{Au}^{3+}$ 与 $\mathrm{Pt}^{4+}$ 的摩尔数计算), 密封无菌于冰箱 $4{ }^{\circ} \mathrm{C}$ 保存备用. 金铂纳米合金聚集体(GPA)制备: 取 $2.0 \mathrm{~mL}$ $56.0 \mu \mathrm{g} / \mathrm{mL} \mathrm{GP}$ 于试管中, 加入 $0.5 \mathrm{~mL} 2.0 \mathrm{~mol} / \mathrm{L} \mathrm{KCl}$ 溶 液, 混匀后溶液呈灰色, 其浓度为 $44.8 \mu \mathrm{g} / \mathrm{mL}$.

\section{4 免疫金铂探针的制备}

\subsection{1 纳米金铂溶液 $\mathrm{pH}$ 值的选择}

本文考察了不同 $\mathrm{pH}$ 对于纳米金铂标记的影响. 依 次在 $5 \mathrm{~mL}$ 具塞刻度试管中加入 $500 \mu \mathrm{L} 56.0 \mathrm{mg} / \mathrm{L}$ (以 $\mathrm{Au}$ 计)金铂纳米溶液, 一定量的 $0.1 \mathrm{~mol} / \mathrm{L} \mathrm{HCl}$ 或 $0.1 \mathrm{~mol} / \mathrm{L}$ $\mathrm{K}_{2} \mathrm{CO}_{3}$ 将纳米金铂溶液 $\mathrm{pH}$ 调节至 $2.5 \sim 9.0$, 再分别加入 $150 \mu \mathrm{L} 2 \mu \mathrm{g} / \mathrm{mL}$ 的兔抗 $\mathrm{hCG}$ 溶液, 放置 $10 \mathrm{~min}$, 加入 10 $\mu \mathrm{L} 10 \% \mathrm{KCl}$, 混匀, 放置 $2 \mathrm{~h}$, 稀释至 $2 \mathrm{~mL}$, 测定共振

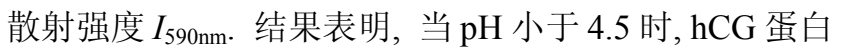
质不能稳定纳米金铂, 共振散射强度值较大; 当 $\mathrm{pH}$ 大 于 4.5 时, $I_{590 \mathrm{~nm}}$ 较小且趋于稳定(表 3). 有研究文献报道: 当纳米金标记蛋白质时 $\mathrm{pH}$ 应等于或高于 0.5 等电点以 上时两者才能牢固结合. 本实验中标记纳米金铂的 $\mathrm{pH}$ 也是高于 $\mathrm{hCG}$ 的等电点 2.95 , 两者的结合才趋于稳定, 此时 $\mathrm{hCG}$ 蛋白质包裹了纳米金铂, $I_{590 \mathrm{~nm}}$ 较小且趋于稳 定. 故本文选择 $\mathrm{pH}$ 值 6.0 .

\subsection{2 兔抗 hCG 用量的选择}

依次在 $5 \mathrm{~mL}$ 具塞刻度试管中加入 $500 \mu \mathrm{L} 56.0$ $\mathrm{mg} / \mathrm{L}$ (以 $\mathrm{Au}$ 计) 金铂纳米溶液, 调节 $\mathrm{pH}$ 为 6.0 , 分别加
入 $0,50,80,100,150,200,250,300,350,400,450 \mu \mathrm{L} 2$ $\mu \mathrm{g} / \mathrm{mL}$ 的兔抗 $\mathrm{hCG}$ 溶液, $10 \mathrm{~min}$ 后加入 $10 \mu \mathrm{L} 10 \% \mathrm{KCl}$, 混匀, 放置 $2 \mathrm{~h}$, 稀释至 $2.0 \mathrm{~mL}$, 测定共振散射强度 $I_{590 \mathrm{~nm}}$. 结果表明, 当 $\mathrm{hCG}$ 的量大于 $150 \mu \mathrm{L}$ 时, $I_{590 \mathrm{~nm}}$ 较 小且趋于不变(表 4), 故本文选择 $150 \mu \mathrm{L}$.

\subsection{3 兔抗 hCG 纳米金铂(GP-RhCG) 的标记}

取 $10 \mathrm{~mL} 56.0 \mu \mathrm{g} / \mathrm{mL}$ (以 $\mathrm{Au}$ 计)金铂纳米粒子溶液, 加入 $200 \mu \mathrm{L} 0.1 \mathrm{~mol} / \mathrm{L}$ 的 $\mathrm{K}_{2} \mathrm{CO}_{3}$ 调节 $\mathrm{pH}$ 值约为 6.0 , 混 合均匀, 加入 $3.0 \mathrm{~mL} 2 \mu \mathrm{g} / \mathrm{mL}$ 的兔抗 $\mathrm{hCG}$ 溶液, 混合均 匀 $2 \mathrm{~h}$ 后, 于 $4{ }^{\circ} \mathrm{C}$ 保存待用, 其浓度为 $42.4 \mu \mathrm{g} / \mathrm{mL}$ GP-RhCG.

\section{5 实验方法}

在 $5.0 \mathrm{~mL}$ 的刻度试管中，依次移取 $250 \mu \mathrm{L} \mathrm{pH} 5.8$ 磷酸氢二钠-柠檬酸缓冲溶液, $300 \mu \mathrm{L} 42.4 \mu \mathrm{g} / \mathrm{mL}$ GP-RhCG 溶液, $100 \mu \mathrm{L} 1.0 \mu \mathrm{g} / \mathrm{mL} \mathrm{hCG}$ 溶液, 超声 15 $\min$, 加入 $15 \mu \mathrm{L} 10 \% \mathrm{KCl}$, 稀释至 $1.5 \mathrm{~mL}$, 混匀, 此为 免疫反应液. 设置 PMT 电压为 $450 \mathrm{~V}$, 激发和发射狭缝 均为 $5 \mathrm{~nm}$, 发射过滤器衰减为 $1 \% \mathrm{~T}$, 以及激发波长等 于发射波长 $\left(\lambda_{\mathrm{ex}}-\lambda_{\mathrm{em}}=\Delta \lambda=0\right)$ 条件, 用荧光分光光度计 记录其共振散射光谱, 测量 $590 \mathrm{~nm}$ 波长处的共振散射 光强度 $I_{590 \mathrm{~nm}}$; 不加 hCG 做空白, 测量其空白值 $\left(I_{590 \mathrm{~nm}}\right)_{0}$, 计算 $\Delta I_{590 \mathrm{~nm}}=\left(I_{590 \mathrm{~nm}}\right)_{0}-I_{590 \mathrm{~nm}}$.

在 $5.0 \mathrm{~mL}$ 的刻度试管中, 依次移取 $30 \mu \mathrm{L} 0.20$ $\mathrm{mol} / \mathrm{L} \mathrm{CuSO}_{4}$ 溶液, $150 \mu \mathrm{L} 1.23 \mathrm{~mol} / \mathrm{L} \mathrm{KNaC}_{4} \mathrm{H}_{4} \mathrm{O}_{6}, 150$ $\mu \mathrm{L}$ 免疫反应液(稀释 50 倍), $200 \mu \mathrm{L} 5.56 \mathrm{mmol} / \mathrm{L}$ 葡萄糖 溶液, 定容至 $3.0 \mathrm{~mL}$, 混匀, 在 $70{ }^{\circ} \mathrm{C}$ 水浴反应 $7 \mathrm{~min}$, 冰水快速冷却至室温. 设置 PMT 电压为 $500 \mathrm{~V}$, 激发和 发射狭缝均为 $2.5 \mathrm{~nm}$, 发射过滤器衰减为 $1 \% \mathrm{~T}$, 以及激 发波长等于发射波长 $\left(\lambda_{\mathrm{ex}}-\lambda_{\mathrm{em}}=\Delta \lambda=0\right)$ 条件，用荧光分 光光度计记录其共振散射光谱，测量 $610 \mathrm{~nm}$ 波长处的 共振散射光强度 $I_{610 \mathrm{~nm}}$; 不加 $\mathrm{hCG}$ 做空白, 测量其空白 值 $\left(I_{610 \mathrm{~nm}}\right)_{0}$, 计算 $\Delta I_{610 \mathrm{~nm}}=I_{610 \mathrm{~nm}}-\left(I_{610 \mathrm{~nm}}\right)_{0}$.

表 3 不同 $\mathrm{pH}$ 值对金铂纳米标记抗体的影响

Table 3 Effect of $\mathrm{pH}$ value on the labeling

\begin{tabular}{|c|c|c|c|c|c|c|c|c|c|c|c|c|c|c|}
\hline $\mathrm{pH}$ & 2.5 & 3.0 & 3.5 & 4.0 & 4.5 & 5.0 & 5.5 & 6.0 & 6.5 & 7.0 & 7.5 & 8.0 & 8.5 & 9.0 \\
\hline$I_{590 \mathrm{~nm}}$ & 364.3 & 591.5 & 762.6 & 746.9 & 54.8 & 84.1 & 63.0 & 59.1 & 81.8 & 40.3 & 45.8 & 83.8 & 92.7 & 54.7 \\
\hline
\end{tabular}

表 4 兔抗 HCG 用量对金铂纳米标记的影响

Table 4 Effect of RhCG amount on the labeling

\begin{tabular}{cccccccccccc}
\hline $\mathrm{hCG}(\mu \mathrm{L})$ & 0 & 50 & 80 & 100 & 150 & 200 & 250 & 300 & 350 & 400 & 450 \\
\hline$I_{590 \mathrm{~nm}}$ & 233.7 & 224.4 & 121.4 & 166.4 & 72.8 & 80.2 & 82.6 & 59.0 & 49.0 & 50.9 & 60.8 \\
\hline
\end{tabular}

\section{References}

[1] Sanvicens, N.; Marco, M. P. Trends Biotechnol. 2008, 26, 425.

[2] Tongprasert, F.; Wanapirak, C.; Tongsong, T. Prenatal Diagn. 2011, 31,430 .

[3] Cole, L. A.; Butler, S. A.; Khanlian, S. A.; Giddings, A.; Muller, C. Y.; Seckl, M. J.; Kohorn, E. I. Gynecol. Oncol. 2006, 102, 151.

[4] Yang, H.-C.; Yuan, R.; Chai, Y.-Q.; Zhuo, Y. Colloids Surf., B 2011, $82,463$.
[5] Chai, R.; Yuan, R.; Chai, Y.-Q.; Ou, C.-F.; Cao, S.-R.; Li, X.-L. Talanta 2008, 74, 1330.

[6] Lim, T. K.; Matsunaga, T. Biosens. Bioelectron. 2001, 16, 1063.

[7] Yang, G.-M.; Yang, X.-Y.; Yang, C.-Y.; Yang, Y.-H. Colloids Surf., A 2011, 389, 195.

[8] Mao, L.; Yuan, R.; Chai, Y.-Q.; Zhuo, Y.; Yang, X. Sens. Actuators B 2010, 149, 226.

[9] Li, R.; Wu, D.; Li, H.; Xu, C.-X.; Wang, H.; Zhao, Y.-F.; Cai, Y.-Y.; Wei, Q.; Du, B. Anal. Biochem. 2011, 414, 196.

[10] Kim, H. S.; Pyun, J. C. Procedia Chem. 2009, 1, 1043. 
[11] Luo, L.; Zhang, Z.-J.; Hou, L.-Y.; Wang, J.-L.; Tian, W. Talanta 2007, 72, 1293.

[12] Yan, X.; Huang, Z.-B.; He, M.; Liao, X.-M.; Zhang, C.-Q.; Yin, G.-F.; Gu, J.-W. Colloids Surf., B 2012, 89, 86.

[13] Nakamura, N.; Lim, T. K.; Jeong, J. M.; Matsunaga, T. Anal. Chim. Acta 2001, 439, 125.

[14] Hudelist, G.; Wuelfing, P.; Czerwenka, K.; Knöfler, M.; Haider, S.; Fink-Retter, A.; Gschwantler-Kaulich, D.; Pfeiler, G.; Kubista, E.; Singer, C. F. J. Cancer Res. Clin. Oncol. 2009, 135, 191.

[15] Jiang, Z.-L.; Zou, M.-J.; Liang, A.-H. Clin. Chim. Acta 2008, 387, 24.

[16] Liang, A.-H.; Zou, M.-J.; Jiang, Z.-L. Talanta 2008, 75, 1214.

[17] Nakamura, K.; Satomura, S.; Matsuura, S. Anal. Chem. 1993, 65, 613.

[18] Lv, M.-X.; Zhang, J.; Shen, Q.-Y.; Chen, K.-M.; Feng, G.-Y. Chin. J. Biomed. Eng. 1992, 11, 174. (吕鸣详, 张杰, 申群英, 陈昆明, 冯 光易，中国生物医学工程学报, 1992, 11, 174.)

[19] Robinson, G. A.; Hill, H. A.; Philo, R. D.; Gear, J. M.; Rattle, S. J.; Forrest, G. C. Clin. Chem. 1985, 31, 1449.

[20] Guo, X.-Y.; Guan, Y.-P.; Yang, B.; Wang, Y.-N.; Lan, H.-L.; Yang, Z.-H.; Lu, Z.-H. Int. J. Mol. Sci. 2006, 7, 274.

[21] Vaitukatitis, J. L.; Braunstein, G. D.; Ross, G. T. Am. J. Obstet. Gynecol. 1972, 113, 751.
[22] Tung, N. H.; Chikae, M.; Ukita, Y.; Viet, P. H.; Takamura, Y. Anal. Chem. 2012, 84, 1210.

[23] Liang, A.-H.; Huang, Y.-J.; Jiang, Z.-L. Clin. Chim. Acta 2007, 383, 73.

[24] Lin, S.-M.; Fan, Y.-Y.; Jiang, Z.-L.; Wen, G.-Q.; Liu, Q.-Y.; Liang, A.-H. Acta Chim. Sinica 2010, 68, 339. (凌绍明，范燕燕，蒋治良, 温桂清, 刘庆业, 梁爱惠, 化学学报, 2010, 68, 339.)

[25] Jiang, Z.-L.; Sun, S.-J.; Liang, A.-H.; Huang, W.-X.; Qin, A.-M. Clin. Chem. 2006, 52, 1389.

[26] Li, J.-F.; Jiang, Z.-L.; Deng, A.-P. Chin. J. Chem. 2008, 26, 2297.

[27] Jiang, Z.-L.; Fan, Y.-Y.; Liang, A.-H.; Wen, G.-Q.; Liu, Q.-Y.; Li, T.-S. Plasmonics 2010, 5, 375.

[28] Jiang, C.-N.; Liang, A.-H.; Jiang, Z.-L. Acta Chim. Sinica 2011, 69, 714. (蒋彩娜, 梁爱惠, 蒋治良, 化学学报, 2011, 69, 714.)

[29] Zhang, Y.; Liang, A.-H.; Zhou, L.-P.; Qin, H.-M.; Ouyang, H.-X.; Wang, P.-F.; Jiang, Z.-L. Acta Chim. Sinica 2011, 69, 2153. (张轶, 梁爱惠, 周莲平, 覃惠敏, 欧阳辉祥, 王鹏飞, 蒋治良, 化学学 报, 2011, 69, 2153.)

[30] Jiang, Z.-L.; Huang, Y.-J.; Liang, A.-H.; Pan, H.-C.; Liu, Q.-Y. Biosens. Bioelectron. 2009, 24, 1674.

[31] Jiang, Z.-L.; Liao, X.-J.; Deng, A.-P.; Liang, A.-H.; Li, J.-S.; Pan, H.-C.; Li, J.-F.; Wang, S.-M.; Huang, Y.-J. Anal. Chem. 2008, 80, 8681 .

(Cheng, B.; Fan, Y.) 